\title{
Characterization of bacterial community in the rumen of bovine with laminitis using high- throughput sequencing
}

Jian Guo

Jilin University

Bo Liu

Inner Mongolia Agriculture University: Inner Mongolia Agricultural University

Tuniyazi. Maimai

Jilin University

Caijun Zhao

Jilin University

Yongguo Cao

Jilin University

Naisheng Zhang

Jilin University

Xiaoyu Hu

Jilin University

Yunhe Fu ( $\square$ fuyunhesky@163.com )

Jilin University https://orcid.org/0000-0002-3258-2610

Research article

Keywords: ruminal microbiota, bovine laminitis, LPS, lactic acid

Posted Date: December 8th, 2020

DOI: https://doi.org/10.21203/rs.3.rs-31535/v2

License: (1) (1) This work is licensed under a Creative Commons Attribution 4.0 International License.

Read Full License 


\section{Abstract}

Background: Laminitis- an inflammation of lamella, could cause great economic loss to dairy industry, which has attracted wide attention around the world. Although previous researches explored risk factors of laminitis, the exact pathological mechanism of laminitis is still remaining unclear. In recent years, microbiota is considered as one of the vital parts that played significant role in various diseases processes. However, current studies are far from sufficient. Aim of this study is to explore the characteristics of ruminal microbiota in laminitis cows.

Results: The serum of bovines with or without laminitis from farms was collected to detect concentrations of LPS, lactic acid, and histamine, and the ruminal fluid was collected for 16S rRNA sequence analysis. The results showed that there was a significant increase in LPS and lactic acid levels in laminitis group comparing to control group cows. In addition, the higher abundance of bacteria that riches acid-enhancing metabolites, namely, Candidatus Saccharimonas, Saccharofermentans, Erysipelotrichaceae UCG-009, Erysipelotrichaceae UCG-008, Clostridium papyrosolvens and Ruminococcaceae bacterium AE2021 were detected in the rumen fluid from laminitis bovines.

Conclusions: This article confirmed that difference of rumen microbiota were occurred in rumen between health and laminitis bovines. The elevated abundance of bacteria that riches acid-enhancing metabolites, as well as increased the concentration of lactic acid and LPS could be harmful factors to bovines and increase risks of laminitis.

\section{Introduction}

In the dairy industry, metabolic diseases are main health problems, such as foot disease[1]. Laminitis, an aseptic inflammation of the sensitive lamina of the hoof[2], is one of the key causes to major economic losses[3] and $62 \%$ of which contributed by lameness cases[4]. In bovines, it can be categorized as acute, subacute, chronic and subclinical laminitis according to the severity and duration of the incident[5]. In the stage of acute and subacute laminitis, the cows are crippled in all limbs[3]. However, there are only changes in claws in chronic phase without any obvious systemic symptoms. The hoof is elongated into a flattened and broadened shape, named "slipper foot"[6-8]. Subclinical laminitis was discovered in 1979. Changes in postures and locomotion are insignificant and also occurs after two to three months of sole ulcers and white-line disease $[9,10]$. Although laminitis has been attracting enough attention, its physiopathology remains to be unelucidated[11]. Currently, it is commonly considered as the result of the interaction of various factors, including breeding conditions and metabolic insults, which increases accumulation of toxic substances, such as histamine, lactic acid and LPS[12] leading to vascular lesions and degradation of the suspensory apparatus of the third phalanx within the digit $[4,13,14]$.

Host-rumen-microbe interactions play a critical role in the maintenance of physiological activity so as to be a contributing factor to affect the health of dairy cows[15]. Now, it is well established that the disturbance of ruminal microbiota can induce bovine laminitis through indigestion caused by ruminal 
acidosis. Overuse of the concentrates aiming at increasing the milk yield contributed to the imbalance of ruminal flora. Increasing harmful bacteria could produce more endotoxins and trigger release of histamine and lactic acid[16-19]. Inflammation reactions are main pathological changes in incipient stage of laminitis and the effects of LPS on it have been examined[20]. In addition, previous research showed that intradermal injection of LPS into bovine induced laminitis[21]. In ruminal acidosis, the release of vasoactive substances (LPS and histamine) leads to vasoconstriction and dilation resulting in the destruction of microvasculature of the corium $[22,23]$. The corium- rich in vascular and neuronsnourishes the dermal lamellae between the lamina and the distal phalanx[24, 25]. Although existing researches showed a link between laminitis and lactic acidosis due to carbohydrate overload, we failed to clarify the correlation ship between ruminal microbiota and laminitis. The aim of this paper is to detect the characteristics of rumen flora in cows with laminitis, so as to provide a reference for the development of microecological preparations for treating cow laminitis.

\section{Results}

\section{LPS, Lactic Acid, and Histamine in Serum}

As shown in Fig. 1A-C, the concentration of LPS and lactic acid in laminitis group significantly increased, while there was no difference in histamine in serum.

\section{The Composition of Ruminal Bacterial Community}

PCR was used to test the V4 region of 16S ribosomal RNA (rRNA) gene of ruminal bacteria and identify characteristics of ruminal bacterial community from sixteen ruminal fluid samples ( 8 from control and 8 from laminitis). Rarefaction curves displayed that the majority of bacterial diversity had sufficient sequences proved by the sampling depth (see supplementary Fig. S1). Comparing control with laminitis group, there was no significant difference in community richness and diversity observed by species, Chao 1 , and ace and Shannon index and Simpson index. Interestingly, the nonmetric multidimensional scaling (NMDS) ordination showed that there was a separation of bacterial community between control and laminitis group using the Bray-Curtis dissimilarity (the value of stress was 0.104) (Fig. 2).

\section{Changes of Ruminal Bacterial Community at the Phylum Level}

At the phylum level, these bacterial sequences obtained from all the bovine comprised 21 phyla and shared the same in both groups (see supplementary Table 1). Among them, Bacteroidetes (control vs laminitis, $50.64 \%$ vs $42.28 \%$ ) and Firmicutes (34.85\% vs $43.64 \%$ ) were the most abundant phyla in ruminal bacterial community. In addition, the relative abundance of Proteobacteria ( $8.03 \%$ vs $2.66 \%$ ), Spirochaetes (1.08\% vs $0.48 \%)$, Tenericutes (1.29\% vs $3.65 \%)$, Euryarchaeota $(0.86 \%$ vs $1.66 \%)$, and Cyanobacteria $0.97 \%$ vs $0.95 \%$ ) was about $1 \%$ and more in bovine ruminal microbiota, respectively (As shown in Fig. 3A and Supplementary Table 1). The results of T-test showed that the relative abundance of Tenericutes, Saccharibacteria, and SR1 (Absconditabacteria) significantly increased in bovine with laminitis than control group (As shown in Fig. 3B). 


\section{Changes of Ruminal Bacterial Community at the Genus and Species Levels}

At the genus level, these bacterial sequences detected from all the animals comprised 245 genera. The results obtained from preliminary analysis of the dominant genera are displayed in Fig. 4A and supplementary table 2, Prevotella 1 (control vs laminitis, $20.44 \%$ vs $13.46 \%)$, Succiniclasticum $(7.77 \%$ vs $7.06 \%$ ), Succinivibrionaceae UCG-002 (4.34\% vs $0.79 \%$ ), Christensenellaceae R-7-group (4.63\% vs $7.94 \%$ ), Succinivibrionaceae UCG-001 (2.20\% vs 0.30\%), Ruminococcaceae NK4A214-group (3.52\% vs $5.11 \%$ ), Ruminococcaceae UCG-014 (2.12\% vs 3.29\%) and Rikenellaceae mRC9 gut group (3.85\% vs $4.24 \%$ ). Ttest showed that the relative abundance of Candidatus Saccharimonas, Saccharofermentans, and Erysipelotrichaceae UCG-009 significantly increased in laminitis group. Meanwhile, none of differences of the other genera was statistically significant (Fig. 4B).

At the species level, the T-test analysis presented that species of Clostridium papyrosolvens and Ruminococcaceae bacterium AE2021 significantly increased while the relative abundance of Prevotella ruminicola, Prevotella sp. CA17 and Treponema saccharophilum significantly decreased in ruminal microbiota from laminitis samples (Fig. 5A-E). Furthermore, the core genera shared by healthy and laminitis bovine were used to evaluate the link between unique bacterial microbiota and laminitis. The Venn diagram illustrated that the proportion of main genera of healthy group was close to $90.66 \%$ and that of laminitis group was responsible for $92.74 \%$ (Fig. 5F). These results may explain the relatively positive correlation between changes in bacterial abundance and interactions among shared bacteria and bovine laminitis. The biomarker analysis by linear discriminant analysis (LDA) effect size (LEfSe) and a cladogram generated from LEfSe analysis on microbiota community of rumen to prove that theory. At the genus levels, the obvious biomarkers were Ruminococcaceae UCG 014, Candidatus Saccharimonas, Saccharofermentans and Succinvibrionaceae UCG 002. While that of species levels was no significant biomarkers (Fig. 6A-B).

\section{Discussion}

Bovine laminitis, one of the most cost of lameness conditions, is an economic drain on producers. It is generally accepted that micro-circulation of blood disorder within the corium induced by microbiota metabolites, such as LPS, lactate, and histamine, is the main pathogenesis of laminitis. Thus, in the present study, we detected the characteristics of rumen microbiota and the concentration of LPS, lactic acid, and histamine in the serum from cows between control and laminitis. The results showed that the concentration of LPS and lactate in serum were increased in the laminitis cows compared to the control cows. In addition, the elevated abundance of bacteria, including Candidatus Saccharimonas, Saccharofermentans, Erysipelotrichaceae UCG-009, Erysipelotrichaceae UCG-008, Clostridium papyrosolvens, and Ruminococcaceae bacterium AE2021, that riches acid- enhancing metabolites could

lower the $\mathrm{pH}$ of rumen fluid, which leads to the death of gram-negative bacteria and release of endotoxins in rumen. However, the small samples size limits to some extent the generalization of the findings made in this study. 
LPS is a major component of the outer membrane of gram-negative bacteria. As the main vasoactive substance, LPS plays a key role in inflammatory reactions. When ruminal acidosis occurs, plenty of gramnegative bacteria are dead and LPS release. In the early stage of laminitis, inflammation is the main manifestation. LPS is absorbed into blood circulation through the ruminal wall, and then reaches the micro-circulation of the claw. The local LPS has inflammatory effects, such as the activation of cytokines and acute-phase protein release, thrombocytopenia, leukopenia followed by leukocytosis[26]. These changes would jeopardize claws to induce laminitis[27]. Besides, recent evidence suggested that pathological changes in inflammation could be made by injection of LPS[21]. In this study, the concentration of LPS significantly increased in the laminitis group compared with the healthy group.

Lactic acid, as vasoactive substances as LPS, are associated with laminitis[28]. Both of them can present a risk to claw deterioration and the laminitis process, including vasoconstriction, dilation, edema and thrombosis, and to interfere with the migration and function of defense cells such as neutrophils[28]. Previous studies reported the increase of histamine and lactic acid in bovine serum during laminitis $[4,5$, 28]. Our experiments confirmed previous results and lactic acid in serum of bovine with laminitis went up.

There is an evidence that ruminal bacterial community play a crucial role in pathologic regulation of organism in developing disease[29]. The role of microbial populations received widespread attention across several disciplines in recent years. Therefore, a number of cross-sectional studies suggested an association among microbiota, its metabolites and laminitis. The proportion of phylum Firmicutes and genera Streptococcus and Lactobacillus significantly grew, while the abundance of phyla Bacteroidetes and Fibrobacteres and genera Butyrivibrio and Ruminococcus dramatically declined during the period of laminitis[30]. However, our results showed that there are no significantly difference rumen bacterial phyla between laminitis and control bovines, this may be due to the different diets of different experimental animals. Our study was to collect rumen fluid samples from healthy and laminitis cows with the same diet, whereas other studies have been conducted in animal models of laminitis induced by high carbohydrate feeding.

In the present study, we observed the significant differences in bacterial community between the two groups using NMDS. In brief, compared to healthy group, the genera Candidatus Saccharimonas, Saccharofermentans, Erysipelotrichaceae UCG-009, and Erysipelotrichaceae UCG-008 increased, most of which were associated with fermentable diets and lower ruminal $\mathrm{pH}[31-33]$. With the reduction of $\mathrm{pH}$, plenty of gram-negative bacteria died and released more LPS. At the species level, Clostridium papyrosolvens and Ruminococcaceae bacterium AE2021significantly increased. As Boonsaen P argued, Ruminococcaceae (e.g. Ruminococcus flavefaciens and Ruminococcus albus) could produce lactate[34]. These results suggested alteration of ruminal bacterial community may be a factor inducing laminitis by influencing rumen metabolisms, such as the increase of LPS and lactate.

\section{Conclusion}


In conclusion, this study showed that vasoactive substances associated with laminitis, such as LPS and lactate, increased. Besides, there were differences in ruminal bacterial community between healthy and laminitis bovines. At the genus and species level, the abundance of Candidatus Saccharimonas, Saccharofermentans, Erysipelotrichaceae UCG-009, Erysipelotrichaceae UCG-008, and Clostridium papyrosolvens and Ruminococcaceae bacterium AE2021 enhanced. Briefly, targeting ruminal microbiota may play a vital part in preventing bovine laminitis.

\section{Methods}

\section{Farms and Animals}

A cross-sectional study was carried out at smallholder dairy farms located in Linqu Country, Weifang city, Shandong province, China, between November 2018 and June 2019. At the beginning, animals were selected by lameness examination and divided into health and lameness group. lameness bovines were then further examined by shoeing to identify laminitis[35]. blood samples and rumen fluid were collected respectively with vacutainer tubes with EDTA and with sterile $50 \mathrm{~mL}$ centrifuge tubes by rumen puncture[36]. Then samples were kept at $-80^{\circ} \mathrm{C}$ for microbiota analysis.

\section{Lipopolysaccharide Concentration Detection}

Blood was centrifuged at $4000 \mathrm{rpm}$ for $30 \mathrm{~min}$ at $4{ }^{\circ} \mathrm{C}$ and then the supernatants were transferred into a sterile and depyrogenated glass tube. Then the serum was quantified using a chromogenic endpoint assay (Chinese Horseshoe Crab Reagent Manufactory Co.,Ltd., Xiamen, China) with a minimum detection limit of $0.01 \mathrm{EU} / \mathrm{mL}$ under the manufacturer's instructions.

\section{Lactic Acid and Histamine Concentrations Detection}

Serum was extracted from the blood by centrifuging at $4000 \mathrm{rpm}$ for $30 \mathrm{~min}$ at $4{ }^{\circ} \mathrm{C}$, and then was detected the concentration of lactic acid and histamine using the detection kits according to the manufacturer's instructions (Suzhou feiya Biological Technology, Suzhou, China).

\section{DNA Extraction, Illumina MiSeq Sequencing, Bioinformatics Analyses}

The genome DNA of ruminal fluid was extracted using a CTAB/SDS method. The DNA concentration and purity were detected by $1 \%$ agarose gels. To amply the $16 \mathrm{~S}$ rRNA, barcoded primers (16S V4:515F-806R) targeting the V4 region was used. The PCR reactions were conducted with Phusion ${ }^{\circledR}$ High-Fidelity PCR Master Mix (New England Biolabs). PCR products were mixed in equal ratios and then purified with a Qiagen Gel Extraction Kit (Qiagen, Germany). Sequencing libraries were generated using the TruSeq ${ }^{\circledR}$ DNA PCR-Free Sample Preparation Kit (Illumina, USA). The library quality was evaluated by a Qubit@ 2.0 Fluorometer (Thermo Scientific) and an Agilent Bioanalyzer 2100 system. Finally, the library was sequenced on an Illumina HiSeq 2500 platform, and 250 bp paired-end reads were generated. Bacterial community diversity and richness were analyzed by ACE, Chao 1, the Shannon index, the Simpson index, and the observed species. The distance of bacterial community between control and laminitis was 
evaluated by NMDS of Bray-Curtis dissimilarity. The bacterial taxa differentially between control and laminitis were evaluated by LEfse, and the Venn diagram was conducted to evaluate the numbers of core genera in the ruminal contents from the control and the laminitis group bovine.

\section{Statistical Analysis}

Statistical analysis was arranged by GraphPad Prism 6.01 (GraphPad Software, Inc., San Diego, CA). All data are presented as means \pm SEM. To compare differences between various experimental groups, a one-way ANOVA (Dunnett's t-test) and the two-tailed t-test were used. The $P<0.05$ or $P<0.01$ was considered statistical significance.

\section{Declarations}

\section{Acknowledgments}

Thanks to the Xinhe ranch for supporting the sample collection.

\section{Funding}

This work was supported by a grant from the National Natural Science Foundation of China (Nos. 31772812ه31972749), and China Postdoctoral Science Foundation (2020TQ0120).

\section{Availability of data and materials}

The datasets generated during the current study are available from the corresponding author on reasonable request.

\section{Author's contributions}

Yunhe Fu and Xiaoyu Hu designed and performed the study, and drafted the manuscript. Jian Guo performed the rumen fluid and blood collection, and microbiota analysis, and contributed to writing the manuscript. Bo Liu performed the LPS, lactic acid and histamine analysis. T. Maimai and Caijun Zhao performed the literature review. Yongguo Cao and Naisheng Zhang performed the supervised the research project and reviewed the paper.

\section{Ethics approval and consent to participate}

This study was carried out following the guidelines of the Institutional Animal Care and Use Committee (IACUC) of Jilin University, and we have a permission to collect animals samples from the farm owner.

\section{Consent for publication}

Not applicable

\section{Competing interests}


The authors declare that they have no competing interests.

\section{References}

1. Beane RL, Ram R, Gabillet S, Arar K, Monia BP, Corey DR: Inhibiting gene expression with locked nucleic acids (LNAs) that target chromosomal DNA. Biochemistry 2007, 46(25):7572-7580.

2. Root Kustritz MV, Molgaard LK, Malone E: Curriculum Review and Revision at the University of Minnesota College of Veterinary Medicine. J Vet Med Educ, 44(3):459-470.

3. Thoefner MB, Pollitt CC, Van Eps AW, Milinovich GJ, Trott DJ, Wattle O, Andersen PH: Acute bovine laminitis: a new induction model using alimentary oligofructose overload. Journal of dairy science 2004, 87(9):2932-2940.

4. Nocek JE: Bovine acidosis: implications on laminitis. Journal of dairy science 1997, 80(5):10051028.

5. Vermunt JJ: "Subclinical" laminitis in dairy cattle. N Z Vet J 1992, 40(4):133-138.

6. Baggott DG, Russell AM: Lameness in cattle. Br Vet J 1981, 137(1):113-132.

7. Maclean CW: Observations on laminitis in intensive beef units. Vet Rec 1966, 78(7):223-231.

8. Maclean CW: The long-term effects of laminitis in dairy cows. Vet Rec 1971, 89(2):34-37.

9. Zhang R, Huo W, Zhu W, Mao S: Characterization of bacterial community of raw milk from dairy cows during subacute ruminal acidosis challenge by high-throughput sequencing. J Sci Food Agric 2015, 95(5):1072-1079.

10. Peterse DJ: [Nutrition as a possible factor in the pathogenesis of ulcers of the sole in cattle (author's transl)]. Tijdschr Diergeneeskd 1979, 104(24):966-970.

11. Thoefner MB, Wattle O, Pollitt CC, French KR, Nielsen SS: Histopathology of oligofructose-induced acute laminitis in heifers. Journal of dairy science 2005, 88(8):2774-2782.

12. Heymering HW: 80 causes, predispositions, and pathways of laminitis. Vet Clin North Am Equine Pract 2010, 26(1):13-19.

13. Concha C, Carretta MD, Alarcon P, Conejeros I, Gallardo D, Hidalgo Al, Tadich N, Caceres DD, Hidalgo MA, Burgos RA: Oxidative response of neutrophils to platelet-activating factor is altered during acute ruminal acidosis induced by oligofructose in heifers. J Vet Sci 2014, 15(2):217-224.

14. Singh SS, Murray RD, Ward WR: Gross and histopathological study of endotoxin-induced hoof lesions in cattle. J Comp Pathol 1994, 110(2):103-115.

15. Zeineldin M, Barakat R, Elolimy A, Salem AZM, Elghandour MMY, Monroy JC: Synergetic action between the rumen microbiota and bovine health. Microb Pathog 2018, 124:106-115.

16. Gozho GN, Plaizier JC, Krause DO, Kennedy AD, Wittenberg KM: Subacute ruminal acidosis induces ruminal lipopolysaccharide endotoxin release and triggers an inflammatory response. Journal of dairy science 2005, 88(4):1399-1403. 
17. Pilachai R, Schonewille JT, Thamrongyoswittayakul C, Aiumlamai S, Wachirapakorn C, Everts $H$, Hendriks WH: The effects of high levels of rumen degradable protein on rumen $\mathrm{pH}$ and histamine concentrations in dairy cows. J Anim Physiol Anim Nutr (Berl) 2012, 96(2):206-213.

18. Zhao C, Liu G, Li X, Guan Y, Wang Y, Yuan X, Sun G, Wang Z, Li X: Inflammatory mechanism of Rumenitis in dairy cows with subacute ruminal acidosis. BMC Vet Res 2018, 14(1):135.

19. Lepper PM, Held TK, Schneider EM, Bolke E, Gerlach H, Trautmann M: Clinical implications of antibiotic-induced endotoxin release in septic shock. Intensive Care Med 2002, 28(7):824-833.

20. Belknap JK: Black walnut extract: an inflammatory model. Vet Clin North Am Equine Pract 2010, 26(1):95-101.

21. Boosman R, Mutsaers CW, Klarenbeek A: The role of endotoxin in the pathogenesis of acute bovine laminitis. Vet Q 1991, 13(3):155-162.

22. Mgassa MN, Amaya-Posada G, Hesselholt M: Pododermatitis aseptica diffusa (laminitis) in free range beef cattle in tropical Africa. Vet Rec 1984, 115(16):413-414.

23. Brent BE: Relationship of acidosis to other feedlot ailments. J Anim Sci 1976, 43(4):930-935.

24. Davis-Unger J, Pajor EA, Schwartzkopf-Genswein K, Marti S, Dorin C, Spackman E, Orsel K: Economic impacts of lameness in feedlot cattle. Transl Anim Sci 2017, 1(4):467-479.

25. Kossaibati MA, Esslemont RJ: The costs of production diseases in dairy herds in England. Vet $J$ 1997, 154(1):41-51.

26. Andersen PH: Bovine endotoxicosis--some aspects of relevance to production diseases. A review. Acta Vet Scand Supp/ 2003, 98:141-155.

27. Shearer JK, Stock ML, Van Amstel SR, Coetzee JF: Assessment and management of pain associated with lameness in cattle. Vet Clin North Am Food Anim Pract 2013, 29(1):135-156.

28. Irwin LN, Mitchell GE, Jr., Tucker RE, Schelling GT: Histamine, tyramine, tryptamine and electrolytes during glucose induced lactic acidosis. J Anim Sci 1979, 48(2):367-374.

29. Zeineldin M, Aldridge B, Lowe J: Dysbiosis of the fecal microbiota in feedlot cattle with hemorrhagic diarrhea. Microb Pathog 2018, 115:123-130.

30. Li H, Liu J, Zhu W, Mao S: Intraruminal infusion of oligofructose alters ruminal microbiota and induces acute laminitis in sheep. J Anim Sci 2017, 95(12):5407-5419.

31. Li F, Wang Z, Dong C, Li F, Wang W, Yuan Z, Mo F, Weng X: Rumen Bacteria Communities and Performances of Fattening Lambs with a Lower or Greater Subacute Ruminal Acidosis Risk. Front Microbio/ 2017, 8:2506.

32. OpdahI LJ, Gonda MG, St-Pierre B: Identification of Uncultured Bacterial Species from Firmicutes, Bacteroidetes and CANDIDATUS Saccharibacteria as Candidate Cellulose Utilizers from the Rumen of Beef Cows. Microorganisms 2018, 6(1).

33. Huo W, Zhu W, Mao S: Impact of subacute ruminal acidosis on the diversity of liquid and solidassociated bacteria in the rumen of goats. World J Microbiol Biotechnol 2014, 30(2):669-680. 
34. Boonsaen P, Kinjo M, Sawanon S, Suzuki Y, Koike S, Kobayashi Y: Partial characterization of phylogeny, ecology and function of the fibrolytic bacterium Ruminococcus flavefaciens OS14, newly isolated from the rumen of swamp buffalo. Anim Sci J 2018, 89(2):377-385.

35. Manske T, Hultgren J, Bergsten C: The effect of claw trimming on the hoof health of Swedish dairy cattle. Prev Vet Med 2002, 54(2):113-129.

36. Kleen JL, Hooijer GA, Rehage J, Noordhuizen JP: Rumenocentesis (rumen puncture): a viable instrument in herd health diagnosis. Dtsch Tierarztl Wochenschr 2004, 111(12):458-462.

\section{Figures}



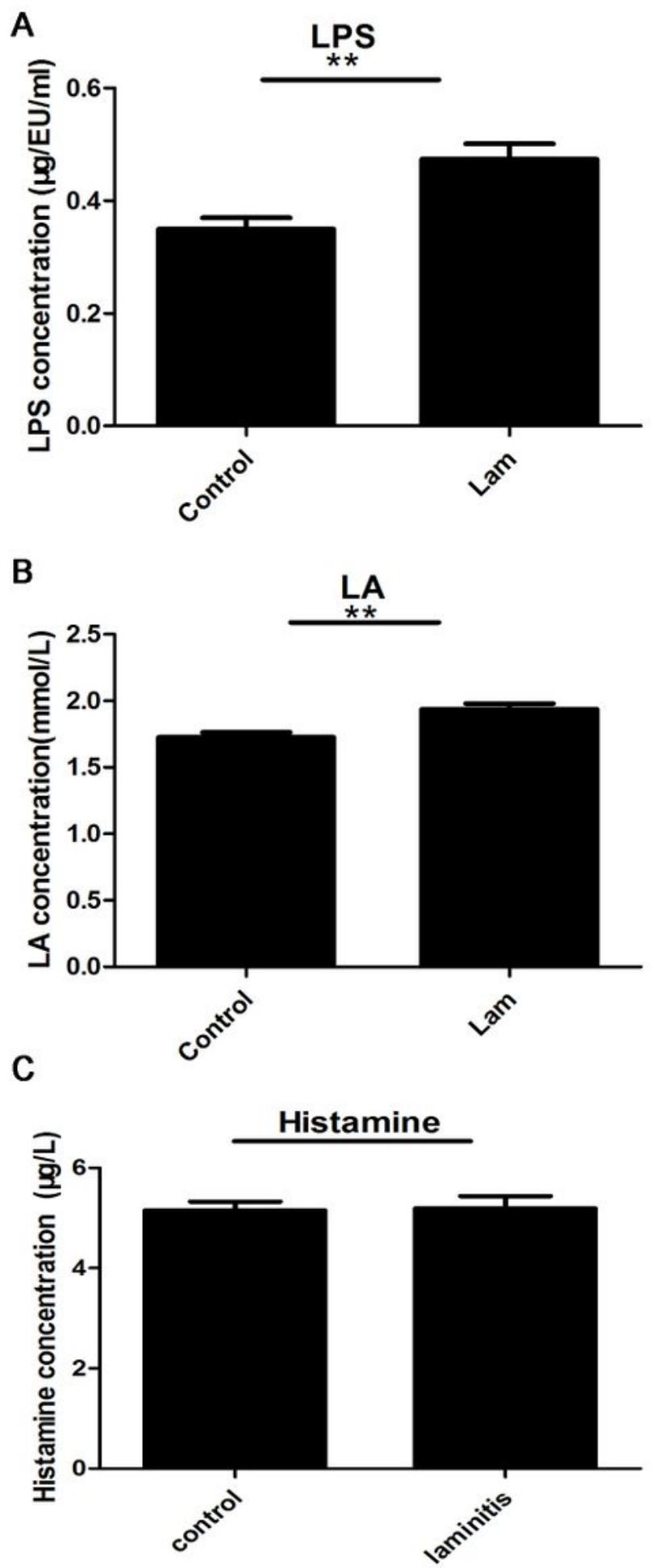

Figure 1

LPS, Lactic Acid, and Histamine in Serum. The concentration of LPS and lactic acid in the laminitis group obviously increase, while that of histamine in serum remained similar. The values presented are the mean \pm SEM. ${ }^{*} \mathrm{P}<0.05$ and ${ }^{*} \mathrm{P}<0.01$ are significantly different from laminitis group. 

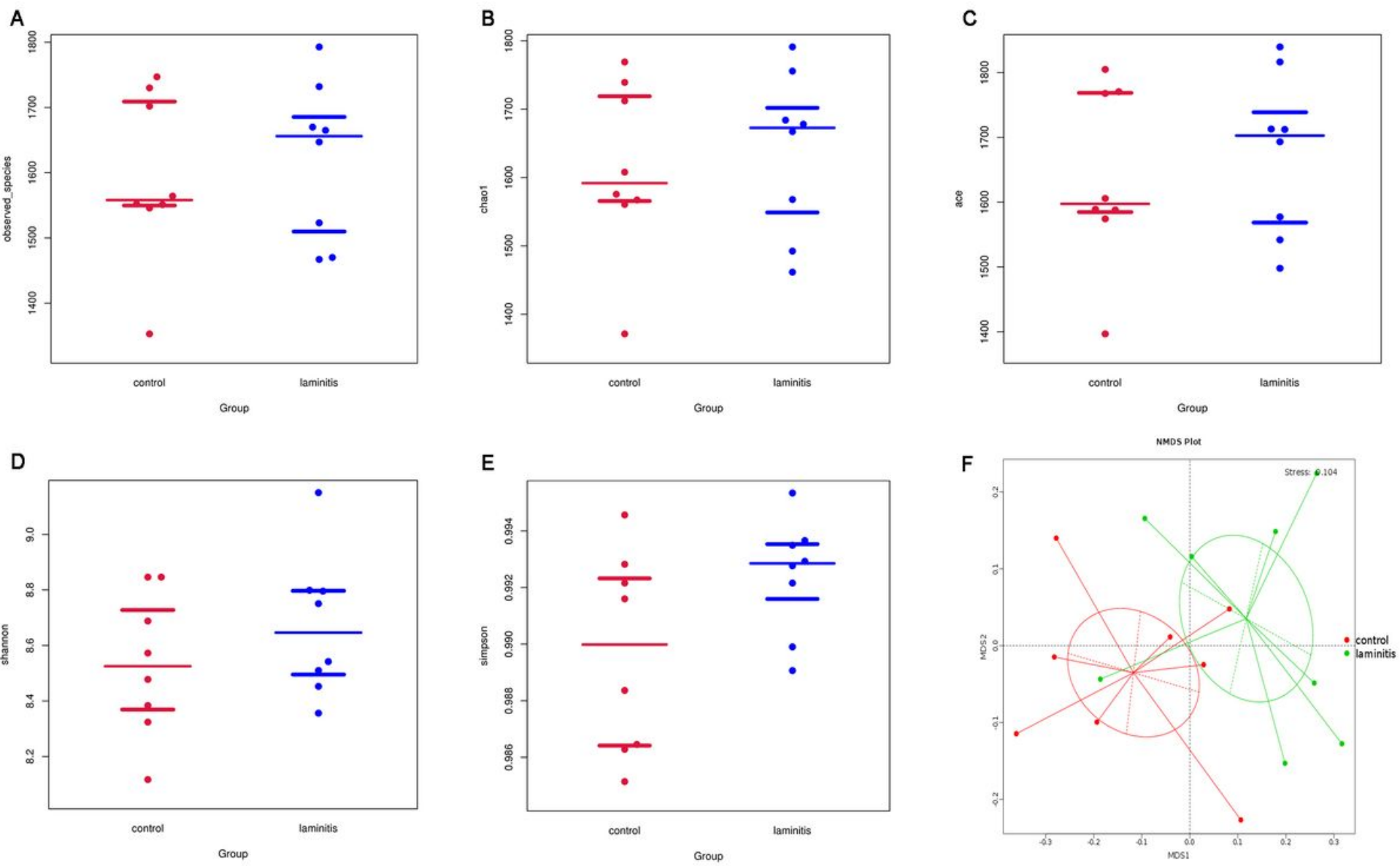

\section{Figure 2}

The Composition of Ruminal Bacterial Community. Differences between healthy and laminitis ruminal microbiota in alpha diversity (A-E). A: observed species, B: Chao 1, C: ace, D: Shannon, E: Simpson. F: Non-metric multidimensional scaling (NMDS) plot of pair wise Bray-Curtis dissimilarities between all samples processed using the two pipelines. Alpha diversity analysis showing no differential abundance between laminitis and healthy rumen microbiome ( $P$ value $>0.05)$.

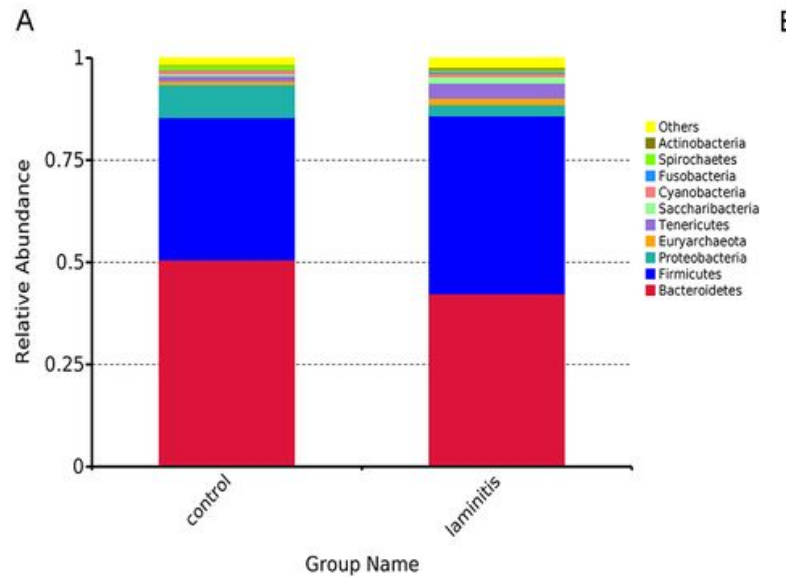

B
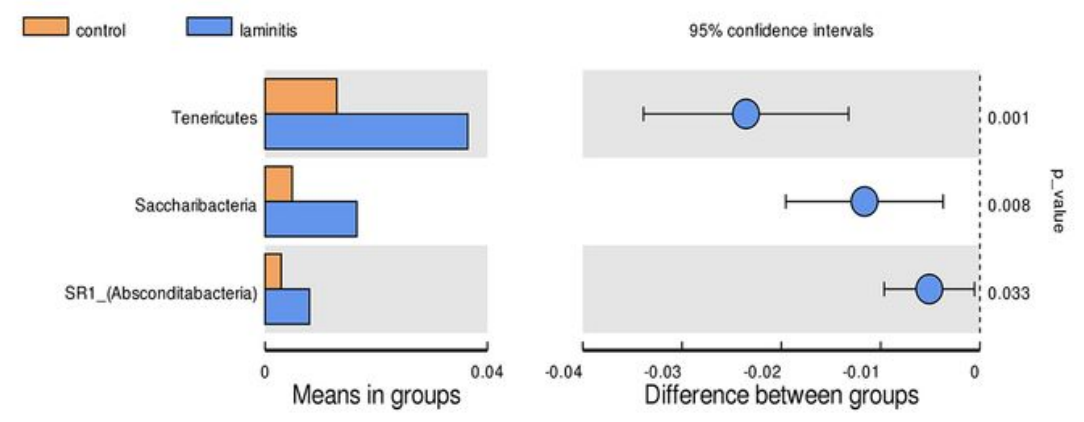

Figure 3 
Changes of Ruminal Bacterial Community at the Phylum Level. Relative abundances of most abundant phylum levels (near $1 \%$ or more in all the sequences) are depicted as mean values for the control and laminitis groups. T-test showing differential abundance between laminitis and healthy ruminal microbiome ( $P$ value $<0.05$ or 0.01 )

A

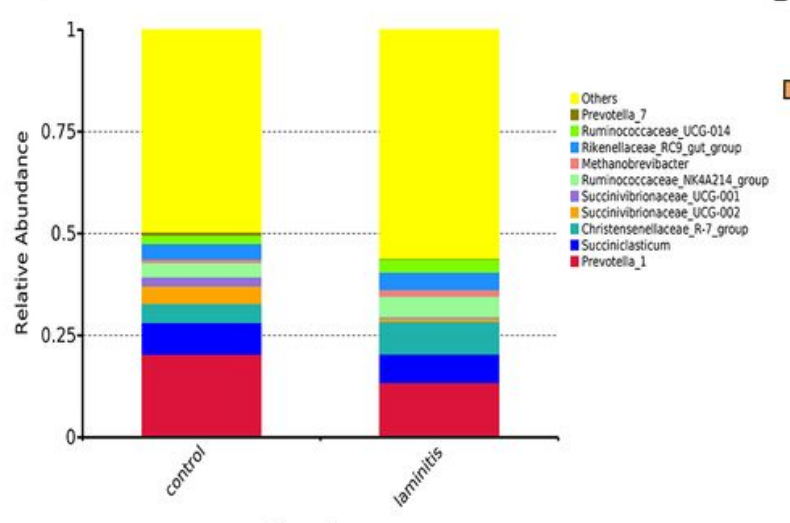

Group Name

B
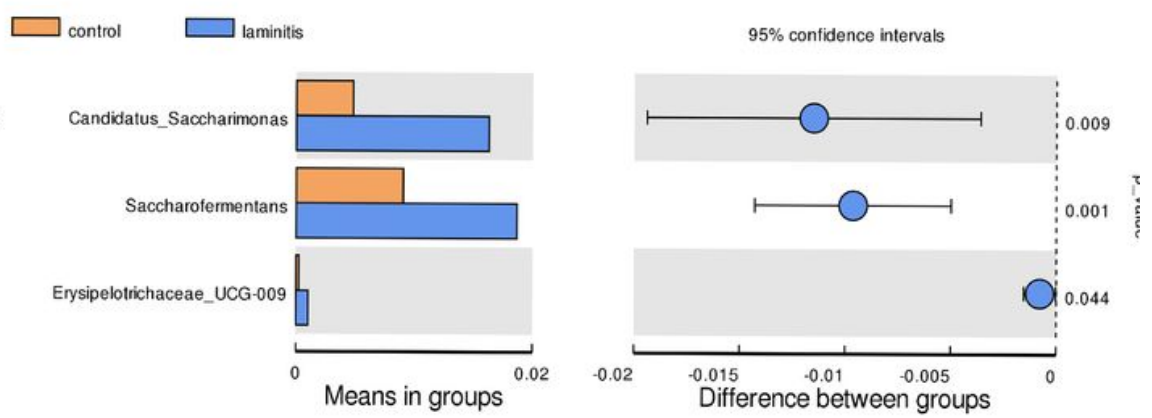

Figure 4

Changes of Ruminal Bacterial Community at the Genus Levels. Relative abundances of most abundant genus levels (near $1 \%$ or more in all the sequences) are depicted as mean values for the control and laminitis groups. T-test showing differential abundance between laminitis and healthy ruminal microbiome ( $P$ value $<0.05$ or 0.01 )

A

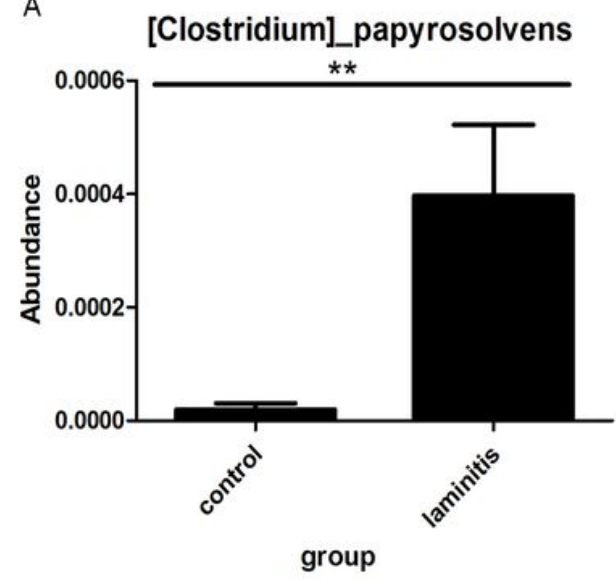

D

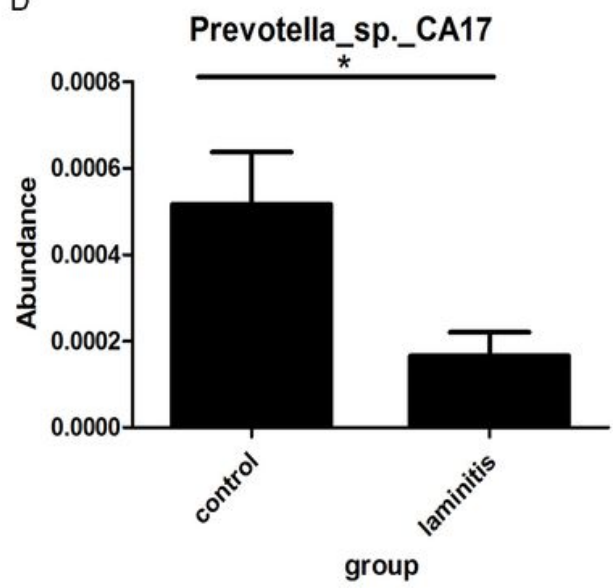

B

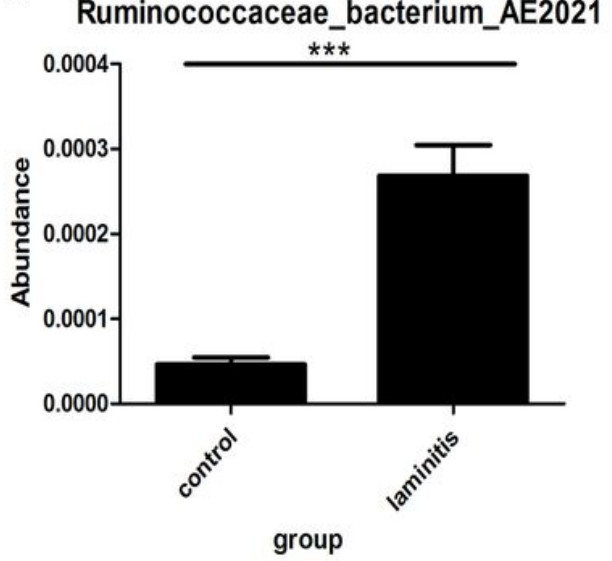

E

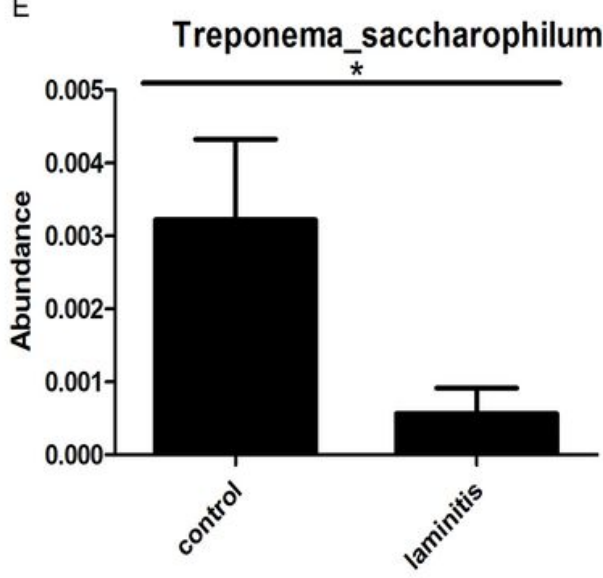

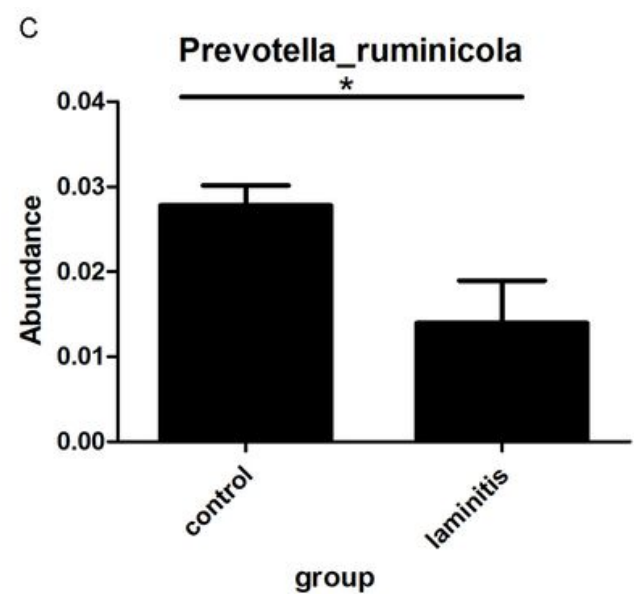

$\mathrm{F}$

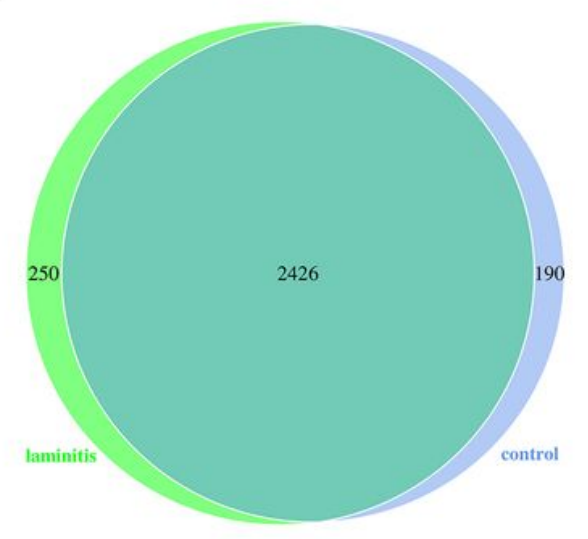




\section{Figure 5}

Changes of Ruminal Bacterial Community at the Species Levels. At the species level, differences between laminitis and healthy rumen microbiota by the two-tailed t-test (A-E). The values presented are the mean \pm SEM. ${ }^{*} \mathrm{P}<0.05$ and ${ }^{*} \mathrm{P}<0.01$ are significantly different from laminitis group. $\mathrm{F}$ : Venn diagram showing the number of core genera in rumen microbiota in laminitis and healthy samples.

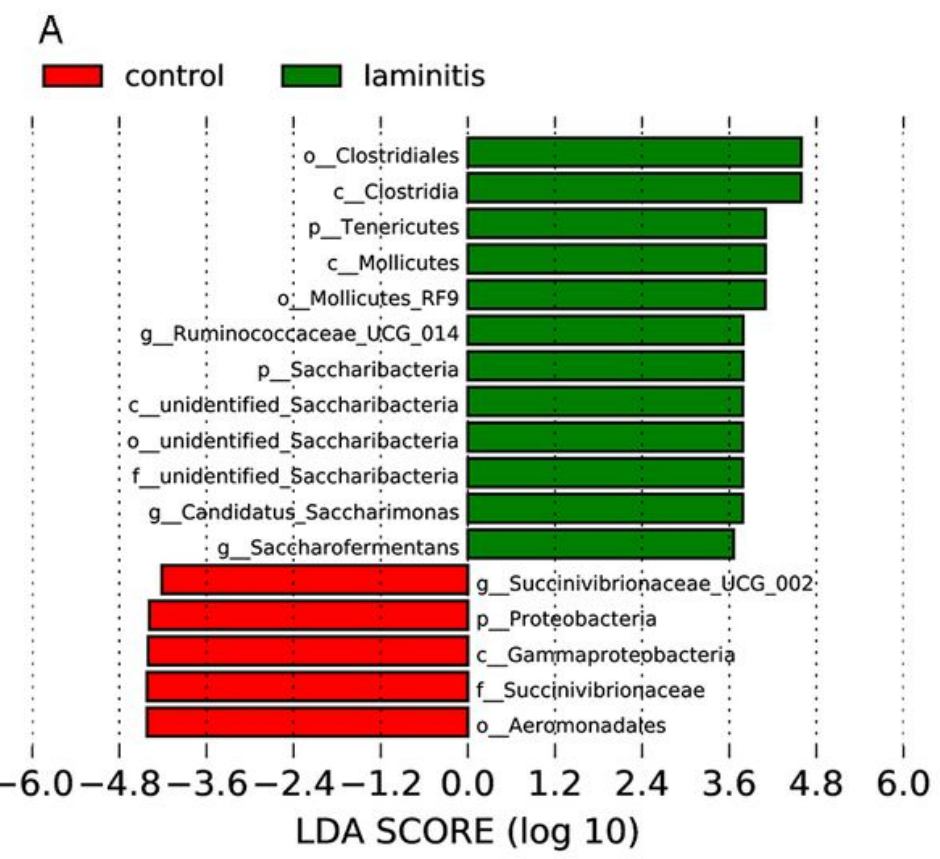

B

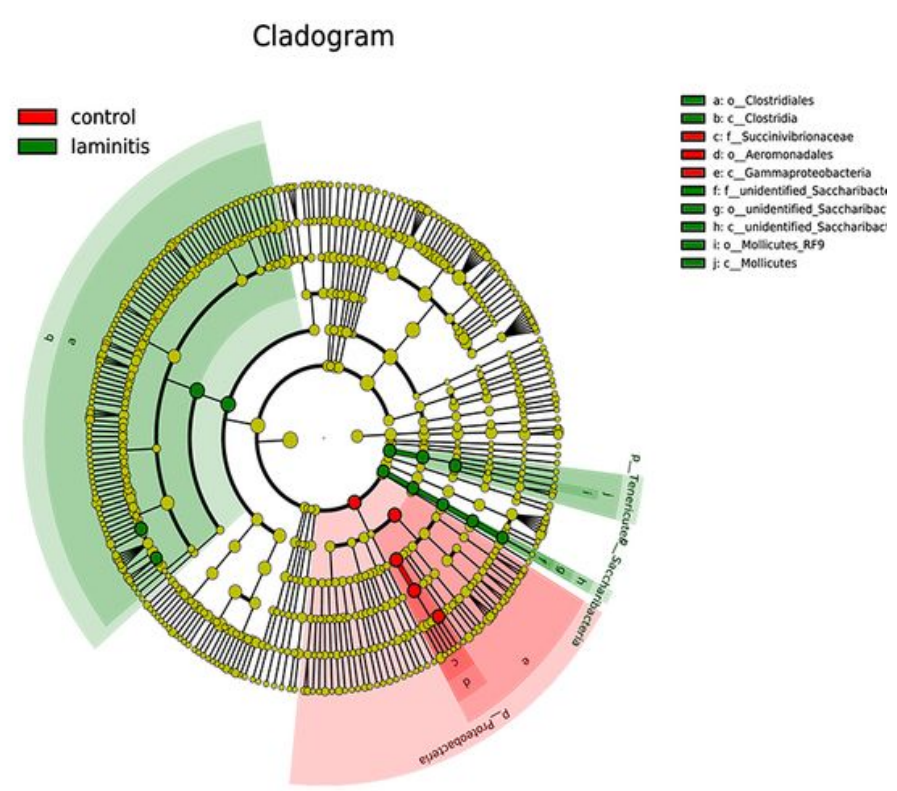

\section{Figure 6}

Taxonomic biomarkers. A: Linear discriminative analysis (LDA) effect size LEfSe analysis between the control (red) and laminitis (green). B: Cardiogram showing differentially abundant taxonomic clades with an LDA score > 3.5 among laminitis and controls, $p$

\section{Supplementary Files}

This is a list of supplementary files associated with this preprint. Click to download.

- Fig.S1.png

- supplementarytable1.xls

- supplementarytable2.xls 